\title{
Note on Loss of Regularity for Solutions of the 3-D Incompressible Euler and Related Equations*
}

\author{
Petre Constantin \\ Department of Mathematics, University of Chicago, Chicago, IL 60637, USA
}

\begin{abstract}
One of the central problems in the mathematical theory of turbulence is that of breakdown of smooth (indefinitely differentiable) solutions to the equations of motion. In $1934 \mathrm{~J}$. Leray advanced the idea that turbulence may be related to the spontaneous appearance of singularities in solutions of the $3-D$ incompressible Navier-Stokes equations. The problem is still open. We show in this report that breakdown of smooth solutions to the $3-D$ incompressible slightly viscous (i.e. corresponding to high Reynolds numbers, or "highly turbulent") Navier-Stokes equations cannot occur without breakdown in the corresponding solution of the incompressible Euler (ideal fluid) equation. We prove then that solutions of distorted Euler equations, which are equations closely related to the Euler equations for short term intervals, do breakdown.
\end{abstract}

\section{Introduction}

The purpose of this paper is twofold: first to discuss the relationship between the breakdown of smooth solutions to incompressible three-dimensional Euler and Navier-Stokes equations; and secondly to present blow-up results for distorted Euler equations.

Both the Navier-Stokes equations and the Euler equations possess local (in time) smooth solutions. Moreover, as the viscosity vanishes the solutions to the Navier-Stokes equations converge uniformly on a short time interval to the solution of the Euler equation [5, 7]. Adapting the method of Kato [5] and using a very simple ODE lemma, we prove in Sect. 1 that as long as the solution to the Euler equation is smooth the solutions to slightly viscous Navier-Stokes equations with the same initial data are smooth.

Sections 2 and 3 are devoted to blow-up results for distorted Euler equations. Differentiating the Euler equations one obtains a quadratic equation for the

* Sponsored by the United States Army under Contract No. DAAG29-80-C-0041, and partially supported by the National Science Foundation under Grant No. MCS-82-01599 
Jacobian matrix of the velocity vector:

$$
\frac{\partial U}{\partial t}+(u \cdot \nabla) U+U^{2}=P,
$$

where $u$ is the velocity vector, $U=\left(\frac{\partial u_{i}}{\partial x_{j}}\right)$ and $P=\left(\frac{\partial^{2} p}{\partial x_{i} \partial x_{j}}\right)$ with $p$ the pressure.

One can use the incompressibility condition $\operatorname{Tr} U=0$ to express $P$ in terms of $U$. Passing to Lagrangian coordinates, the differentiated Euler equations become

$$
\frac{\partial U}{\partial t}+U^{2}+R(t)\left(\operatorname{Tr} U^{2}\right)=0
$$

where $R(t)$ is a matrix of singular integral operators with time varying kernels. What we call the distorted Euler equations are obtained from the above form of the genuine Euler equations by replacing $R(t)$ by $R(0)$ :

$$
\frac{\partial U}{\partial t}+U^{2}+R(0)\left(\operatorname{Tr} U^{2}\right)=0 .
$$

Although these equations are good short time approximations of the Euler equations, the blow-up arguments have no direct bearing on the Euler equations.

In Sect. 2 we discuss the periodic case and we show, by a localization argument reminiscent of the one in [2], that a large class of initial data lead to breakdown of the solution of $(0.2)$. The conditions on the initial data do not involve any largeness assumption but exclude Jacobians. Another drawback in the periodic case is the fact that incompressibility, $\operatorname{Tr} U=0$, is not preserved. This fact is due to the nonvanishing of the mean of $\operatorname{Tr} U^{2}$, but it is not the major reason for the blow-up. (One can modify slightly the equations in order to preserve the constraint $\operatorname{Tr} U=0$ and still prove breakdown.) Moreover, in the whole space case Eqs. (0.2) do preserve incompressibility. Section 3 treats solutions of the distorted Euler equations in the whole space. Foias found [4] that if the initial data for (0.2) have the form

$$
U_{0}(x)=\beta_{0}(|x|)(I-n \pi(x)),
$$

where $n$ is the dimension and $\pi(x)=\left(\frac{x_{i} x_{j}}{|x|^{2}}\right) i, j=1, \ldots, n$, then this form is retained by the solution $U(t, x)$ of $(0.2)$ and leads to a simple equation for the scalar quantity $\beta$. We generalize slightly his result by allowing $U_{0}$ to possess an antisymmetric part, corresponding to the vorticity. We obtain a system of integro-differential equations for two scalar quantities $\beta(t, r)$ (corresponding to the size of the deformation tensor) and $\gamma(t, r)$ corresponding to the modulus of the vorticity. For $\gamma \equiv 0$ we recover the Foias equation. The success of the reduction in the number of variables and unknowns is due to a covariance property of Eq. (0.2) with respect to an action of $O(n)$. We prove breakdown for solutions starting from initial data of the special form

$$
U_{0}(x)=\beta_{0}(|x|)(I-3 \pi(x))+\gamma_{0}(|x|) \frac{x}{|x|} \times .
$$


If one takes the antisymmetric part of the three-dimensional distorted Euler equations and if one identifies $3 \times 3$ antisymmetric matrices $J$ with the vectors given by $J=\omega \times$, one obtains the equation

$$
\frac{\partial \omega}{\partial t}=U \omega
$$

which is the analogue of the vorticity equation for incompressible Euler flows.

In [3] the simple one-dimensional model equation for the three-dimensional vorticity equation

$$
\frac{\partial \omega}{\partial t}=\omega H \omega \quad(H=\text { Hilbert transform })
$$

was suggested. The breakdown of solutions to $(0.2)$ is very similar to that of solutions to $(0.5)$ : The quantity corresponding to the deformation tensor [the symmetric part of $U$ in the case of $(0.2), H \omega$ in the case of $(0.5)]$ becomes infinite in regions when the quantity corresponding to the vorticity (denoted $\omega$ in both cases) is zero.

\section{A Comparison Result}

Let us consider a solution $v$ of the incompressible Euler equations

$$
\left\{\begin{array}{l}
\frac{\partial v}{\partial t}+(v \cdot \nabla) v=\nabla p+f \\
\operatorname{div} v=0 \\
v(0, \cdot)=v_{0}
\end{array}\right.
$$

in either $\mathbb{R}^{3}$ or $T^{3}$ (the three-dimensional torus). In this section we prove that as long as $v(t, \cdot)$ is smooth, the solutions to slightly viscous incompressible NavierStokes equations having $v_{0}$ as initial data are smooth.

We use the notation $H^{m}$ for the Sobolev spaces $H^{m}=H^{m}\left(\mathbb{R}^{3}\right)$ [respectively $\left.H^{m}=H^{m}\left(T^{3}\right)\right]$ and $(\cdot, \cdot)_{m},\|\cdot\|_{m}$ for the corresponding scalar products and norms.

Theorem 1.1. Let $v=v(t, x)$ be a solution of (1.1) for $0 \leqq t \leqq T$, satisfying

$$
\begin{gathered}
\left\|v_{0}\right\|_{m+2}<\infty \quad \text { for some } \quad m \geqq 3 \\
\int_{0}^{T}|\nabla \times v|_{L^{\infty}} d t<\infty
\end{gathered}
$$

Then there exists $v_{0}=v_{0}\left(T ;\left\|v_{0}\right\|_{m+2} ; \int_{0}^{T}|\nabla \times v|_{L^{\infty}} d t\right)$ such that, for every $0<v \leqq v_{0}$ the solution to the Navier-Stokes equation

$$
\left\{\begin{array}{l}
\frac{\partial u}{\partial t}+(u \cdot \nabla) u=v \Delta u+\nabla q+f \\
\operatorname{div} u=0 \\
u(0, \cdot)=v_{0}
\end{array}\right.
$$


is smooth on $[0, T]$. More precisely

$$
\sup _{t \in[0, T]}\|u(t)-v(t)\|_{m} \leqq v \gamma_{m}
$$

for some $\gamma_{m}$ depending on $T,\left\|v_{0}\right\|_{m+2}, \int_{0}^{T}|\nabla \times v|_{L^{\infty}} d t$.

Let us emphasize here that $T$ is not assumed to be small. Instead it is assumed that $v(t)$ belongs to $H^{m+2}$ for $t \in[0, T]$. Indeed, assumption (1.3) was proven by Beale et al. [1] to be a sufficient condition for higher regularity. Their result can be stated as follows

Theorem 1.2 (Beale et al.). Assume $\int_{0}^{T}|\nabla \times v|_{L^{\infty}} d t<\infty$. Let $s \geqq 3, v_{0} \in H^{s}$. There exists a constant $c$ depending on $T, s, \int_{0}^{T}|\nabla \times v|_{L^{\infty}} d t,\left\|v_{0}\right\|_{s}$, such that

$$
\|v(t)\|_{s} \leqq c \quad \text { for } \quad t \leqq T
$$

In order to prove Theorem 1.1 let us consider the difference $w=u-v$. Then $w$ will satisfy

$$
\left\{\begin{array}{l}
\frac{\partial w}{\partial t}-v \Delta w+(v \cdot \nabla) w+(w \cdot \nabla) v+(w \cdot \nabla) w=v \Delta v+\nabla r \\
\operatorname{div} w=0 \\
w(0, \cdot)=0
\end{array}\right.
$$

We take the scalar product of (1.7) with $w$ in $H^{m}, m \geqq 3$ and use

$$
\begin{aligned}
& \left|((v \cdot \nabla) w, w)_{m}\right| \leqq c_{m}\|v\|_{m}\|w\|_{m}^{2}, \\
& \left|(w \cdot \nabla v ; w)_{m}\right| \leqq c_{m}\|v\|_{m+1}\|w\|_{m}^{2}
\end{aligned}
$$

for $v \in H^{m+1}, \operatorname{div} v=0, w \in H^{m+1}, \operatorname{div} w=0$ (see [5]).

Using the fact that $-v(\Delta w, w)_{m} \geqq 0$, we obtain

$$
\frac{d}{d t}\|w\|_{m} \leqq v\|\Delta v\|_{m}+c_{m}\|v\|_{m+1}\|w\|_{m}+c_{m}\|w\|_{m}^{2} .
$$

Let us multiply (1.10) by $\exp \left(-c_{m} \int_{0}^{t}\|v\|_{m+1} d s\right)$ and consider the quantity

$$
y=\|w\|_{m} \exp \left(-c_{m} \int_{0}^{t}\|v\|_{m+1} d s\right)
$$

We obtain the inequality

$$
\left\{\begin{array}{l}
\frac{d y}{d t} \leqq v F(t)+G y^{2} \\
y(0)=0
\end{array}\right.
$$


with

$$
F(t)=\|\Delta v\|_{m} \exp -c_{m} \int_{0}^{t}\|v\|_{m+1} d s
$$

and

$$
G=c_{m} \exp c_{m} \int_{0}^{T}\|v\|_{m+1} d t
$$

We shall make use now of an elementary lemma:

Lemma 1.3. Let $T>0, G>0$ be given constants and let $F(t)$ be a nonnegative continuous function on $[0, T]$. Let $v_{0}$ be defined by

$$
v_{0}=\frac{1}{8 T G \int_{0}^{T} F(t) d t}
$$

Then, for every $0<v \leqq v_{0}$, every solution $y \geqq 0$ of (1.11) is uniformly bounded on $[0, T]$ and

$$
y(t) \leqq \operatorname{Min}\left\{\frac{3}{2 T G}, 12 v \int_{0}^{T} F(t) d t\right\}
$$

Proof of Lemma. Let us define $\varepsilon$ by

$$
\varepsilon=\operatorname{Min}\left\{\frac{1}{4 T^{2} G}, 16 v^{2} G\left(\int_{0}^{T} F(t) d t\right)^{2}\right\} .
$$

We divide (1.11) by $\left(1+\sqrt{\frac{G}{\varepsilon} y}\right)^{2}: \frac{\frac{d y}{d t}}{\left(1+\sqrt{\left.\frac{G}{\varepsilon} y\right)^{2}}\right.} \leqq \nu F+\varepsilon$. We integrate between 0 and $t$ :

$$
\sqrt{\frac{\varepsilon}{G}} \frac{1}{1+\sqrt{\frac{G}{\varepsilon}} y(t)} \geqq \sqrt{\frac{\varepsilon}{G}}-\varepsilon T-v \int_{0}^{T} F(t) d t .
$$

The choice $\varepsilon \leqq \frac{1}{4 T^{2} G}$ implies $\varepsilon T \leqq \frac{1}{2} \sqrt{\frac{\varepsilon}{G}}$ and, for $v \leqq v_{0}$ one has $v \int_{0}^{T} F(t) d t \leqq \frac{1}{4} \sqrt{\frac{\varepsilon}{G}}$. Indeed, if $\varepsilon=16 v^{2} G\left(\int_{0}^{T} F(t) d t\right)^{2}$ the last inequality is an equality and if $\varepsilon=\frac{1}{4 T^{2} G}$, it follows from

$$
\frac{1}{4} \sqrt{\frac{\varepsilon}{G}}=\frac{1}{8 T G}=v_{0} \int_{0}^{T} F(t) d t \geqq v \int_{0}^{T} F(t) d t
$$

Thus (1.17) becomes $\frac{1}{1+\sqrt{\frac{G}{\varepsilon} y(t)}} \geqq \frac{1}{4}$ which implies (1.15). 
We return to the proof of Theorem 1.1 .

We apply Lemma 1.3 to (1.11) with $F, G$ defined in (1.12), (1.13). We find $v_{0}$ depending on $T, m, \int_{0}^{T}\|v\|_{m+2} d t$ such that, if $0<v \leqq v_{0}$ and as long as $w(t)$ belongs to $H^{m+2}, t \leqq T$, one has

$$
\|w(t)\|_{m} \leqq \gamma_{m} v
$$

for some $\gamma_{m}$ depending on $T, m, \int_{0}^{T}\|v\|_{m+2} d t$.

Using standard calculus inequalities one can find bounds of the type

$$
\|w(t)\|_{m+2} \leqq\|v(t)\|_{m+2}+\left[\left\|v_{0}\right\|_{m+2}+\int_{0}^{T}\|f\|_{m+2} d t\right] \exp \left(c_{m} \int_{0}^{T}\|w\|_{m}+\|v\|_{m} d s\right)
$$

Since the validity of (1.18) depends upon $w(t)$ belonging to $H^{m+2}$ but not upon the size of $\|w(t)\|_{m+2}$, one can argue by contradiction and infer that $\|w(t)\|_{m+2}$ cannot become infinite for $t \leqq T$ and that (1.18) is true for all $t \leqq T$. We omit further details.

\section{Distorted Euler Equations}

In this section we prove breakdown of smooth solutions of a "semi-Lagrangian" version of the Euler equations. We start by recalling the Euler equation in $\mathbb{R}^{n}$ or $T^{n}$

$$
\left\{\begin{array}{l}
\partial_{t} u_{i}+u_{j} \partial_{j} u_{i}=\partial_{i} p, \quad j, i=1, \ldots, n \\
\partial_{i} u_{i}=0 \\
u(0, \cdot)=u_{0}(\cdot)
\end{array}\right.
$$

(Here $\partial_{t}=\frac{\partial}{\partial t}, \partial_{j}=\frac{\partial}{\partial x_{j}}$ and summation convention is used.)

Differentiating (2.1) we obtain

$$
\left\{\begin{array}{l}
\partial_{t} U+(u \cdot \nabla) U+U^{2}=P \\
\operatorname{Tr} U=0 \\
U(0, \cdot)=U,
\end{array}\right.
$$

where $U$ is the $n \times n$ matrix $U=\left(\partial_{j} u_{i}\right), i=1, \ldots, n, j=1, \ldots, n$ and $P$ is the Hessian of the pressure $P=\left(\partial_{i j}^{2} p\right), i, j=1, \ldots, n$. The constraint $\operatorname{Tr} U=0$ (incompressibility) is maintained if $\operatorname{Tr} P=\operatorname{Tr} U^{2}$. This means that $p$ solves $\Delta p=\operatorname{Tr} U^{2}$, and therefore the matrix $P$ can be expressed in terms of $U$ :

$$
P=\left(-R_{i} R_{j}\left(\operatorname{Tr} U^{2}\right)\right), \quad i, j=1, \ldots, n,
$$

where $R_{i}$ are the Riesz transforms defined by

$$
R_{i}=(-\Delta)^{-1 / 2} \partial_{i}
$$


Let us denote by $R$ the operator acting on $n \times n$ matrix valued functions $M=\left(m_{i j}\right)$, $i=1, \ldots, n, j=1, \ldots, n$ by

$$
(R M)_{i j}=R_{i} R_{k}\left(m_{k j}\right) .
$$

We identify scalar functions $f$ with the matrices $f \cdot I$, where $I$ is the $n \times n$ identity matrix. The (differentiated) Euler equations can be written as

$$
\left\{\begin{array}{l}
\partial_{t} U+(u \cdot \nabla) U+U^{2}+R\left(\operatorname{Tr} U^{2}\right)=0 \\
\operatorname{Tr} U=0 \\
U(0, \cdot)=U_{0}(\cdot) .
\end{array}\right.
$$

If one passes to Lagrangian coordinates in (2.6), that is, if one uses the change of variables $\alpha \stackrel{\Phi^{t}}{\longmapsto} x(t, \alpha)$ for $x(t, \alpha)$ solving

$$
\left\{\begin{array}{l}
\frac{d x}{d t}=u(t, x) \\
x(0, \alpha)=\alpha,
\end{array}\right.
$$

the Euler equations (2.6) become

$$
\left\{\begin{array}{l}
\partial_{t} V+V^{2}+R(t)\left(\operatorname{Tr} V^{2}\right)=0 \\
\operatorname{Tr} V=0 \\
V(0, \cdot)=V_{0}
\end{array}\right.
$$

where $V(t, \alpha)=U(t, x(t, \alpha))$ is the pullback of $U$ and $R(t)$ is the pullback of $R$ through $\Phi^{t}$ :

$$
R(t) M=\left[R\left(M \cdot\left(\Phi^{t}\right)^{-1}\right)\right] \cdot \Phi^{t} .
$$

More precisely if $k_{i j}(x, y)$ is a kernel for $R_{i} R_{j}$, a kernel for $R(t)_{i j}$ will be

$$
k_{i j}^{t}(\alpha, \beta)=k_{i j}(x(t, \alpha), x(t, \beta)) .
$$

(We used the well-known fact that determinant of Jacobian of $\Phi^{t}$ is one.) At $t=0$ the operator $R(t)$ coincides with the Riesz operators $R(0)=R$; this because $x(0, \alpha)=\alpha$. The distorted Euler equations are obtained from the genuine Euler equations (2.8) by freezing $R(t)$ at $t=0$ :

$$
\left\{\begin{array}{l}
U_{t}+U^{2}+R\left(\operatorname{Tr} U^{2}\right)=0 \\
U(0, \cdot)=U_{0}
\end{array}\right.
$$

Let us note that while (2.10) are valid approximations of (2.8) for a short time, the blow up arguments that we are going to give have no direct bearing on the Euler equations.

Equations (2.10) are well-posed in a variety of spaces. For instance we can consider the Sobolev spaces $\left(H^{s}\right)^{n^{2}}$ of matrices with entries in $H^{s}, s>\frac{n}{2}$. If $s>\frac{n}{2}$ $H^{s}$ are Banach algebras under pointwise multiplication; the operators $R_{j}$ are bounded in $H^{s}$ (for any s, of course). We conclude that, if $U$ is a solution of (2.10),

$$
\frac{d}{d t}\|U\|_{s} \leqq c_{s}\|U\|_{s}^{2}
$$


and the local existence and uniqueness of solutions of $(2.10)$ follow in standard manner.

We shall treat first the periodic case; we seek solutions to (2.10) which satisfy $U\left(x+L e_{i}\right)=U(x)$ for any $e_{i}=(0, \ldots, 1, \ldots, 0)^{t}$ and some $L>0$. We may assume $L=1$ without loss of generality. Alternately, we shall refer to $U$ as being defined on the $n$ dimensional torus $T^{n}=\mathbb{R}^{n} / \mathbb{Z}^{n}$. Let us denote, for a point $x$ in $T^{n}$ by $\Gamma_{i}(x)$ the $i^{\text {th }}$ principal circle passing through $x$ :

$$
\Gamma_{i}(x)=\left\{y \in T^{n} \mid y_{j}=x_{j} \bmod 1, j=1, \ldots, n, j \neq i\right\} ; \quad i=1, \ldots, n .
$$

Let us denote, for a matrix $U$, by $S$ and $J$ the symmetric and respectively antisymmetric parts of $U$ :

$$
S=\frac{U+U^{*}}{2} ; J=\frac{U-U^{*}}{2}, \text { where } U^{*} \text { is the transposed of } U .
$$

Theorem 2.1. Let $U_{0}$ be a smooth $n \times n$ matrix valued function on $T^{n}$ satisfying

(i) $\operatorname{Tr} U_{0}(x)=0$ for all $x \in T^{n}$.

(ii) There exists $x_{0} \in T^{n}$ and $i, 1 \leqq i \leqq n$ such that

and

$$
\operatorname{supp} J_{0} \cap \Gamma_{i}\left(x_{0}\right)=\emptyset \quad\left(J_{0}=\frac{U_{0}-U_{0}^{*}}{2}\right)
$$

$$
\int_{\Gamma_{i}\left(x_{0}\right)} U_{0 i i} d x_{i}<0 \quad \text { (no summation). }
$$

Then the solution of (2.10) having $U_{0}$ as initial data breaks down in finite time. More precisely the symmetric part of the solution $U(t, x)$ becomes infinite near $\Gamma_{i}\left(x_{0}\right)$ in finite time.

Proof. Let us introduce first some notation. We denote for two matrices $M, N$ by $(M ; N)$ the scalar product

$$
(M ; N)=\operatorname{Tr} M N^{*}
$$

For two matrix valued functions on $T^{n}$ we denote by $\langle M, N\rangle$ the scalar product

$$
\langle M, N\rangle=\int_{T^{n}}(M(x) ; N(x)) d x .
$$

Let us first remark that the operator $R$ is symmetric:

$$
\langle R M, N\rangle=\langle M, R N\rangle \text {. }
$$

Let us break (2.10) into its symmetric and antisymmetric parts:

$$
\begin{gathered}
\partial_{t} S+S^{2}+J^{2}+R\left(\operatorname{Tr} U^{2}\right)=0, \\
\partial_{t} J+S J+J S=0 .
\end{gathered}
$$

We deduce from (2.16) that

$$
\operatorname{supp} J(t, \cdot) \subset \operatorname{supp} J_{0} \text {. }
$$


Indeed, we can prove (2.17) by noticing that, for any fixed $x \in T^{n}$,

$$
\frac{1}{2} \frac{d}{d t}(J(t, x) ; J(t, x))=\operatorname{Tr}\left(S J^{2}+J S J\right)=2 \operatorname{Tr} S J^{2} \leqq 2 m(t, x)(J(t, x) ; J(t, x)),
$$

where $m(t, x)$ is the maximum of the absolute values of the eigenvalues of $S(t, x)$. It follows from Gronwall's inequality that, as long as $S(t, x)$ is smooth, if $J_{0}(x)=0$, $J(t, x)=0$. Let $\Phi$ be an $n \times n$ matrix valued smooth function satisfying the following conditions:

(a) $\operatorname{supp} \Phi \cap \operatorname{supp} J_{0}=\emptyset$,

(b) for every $x \in T^{n}, \Phi(x)$ is a symmetric, nonnegative matrix, i.e. $\Phi(x)=\Psi(x)^{2}$ for some symmetric $\Psi(x)$.

(c) $R \Phi=0$.

(d) $\left\langle S_{0}, \Phi\right\rangle<0$.

Let us postpone for the moment the construction of $\Phi$ and proceed with the proof. Taking the scalar product of (2.15) with $\Phi$ we obtain

$$
\frac{d}{d t}\langle S, \Phi\rangle+\left\langle S^{2}, \Phi\right\rangle+\left\langle J^{2}, \Phi\right\rangle+\left\langle R\left(\operatorname{Tr} U^{2}\right), \Phi\right\rangle=0 .
$$

Now $\left\langle R\left(\operatorname{Tr} U^{2}\right), \Phi\right\rangle=\left\langle\operatorname{Tr} U^{2}, R \Phi\right\rangle=0$ because of assumption (c) and of the symmetry (2.14) of $R$. Moreover, combining (2.17) and assumption (a), we deduce $\left\langle J^{2}, \Phi\right\rangle=0$. Thus (2.18) becomes

$$
\frac{d}{d t}\langle S, \Phi\rangle+\left\langle S^{2}, \Phi\right\rangle=0
$$

Now

$$
\begin{aligned}
|\langle S, \Phi\rangle| & =\left|\int_{T^{n}} \operatorname{Tr}(S(x) \Phi(x)) d x\right| \leqq \int_{T^{n}}|\operatorname{Tr}(S(x) \Psi(x) \Psi(x))| d x \\
& \leqq \int_{T^{n}}\left(\operatorname{Tr}(S(x) \Psi(x))(S(x) \Psi(x))^{*}\right)^{1 / 2}(\operatorname{Tr} \Psi(x) \Psi(x))^{1 / 2} d x \\
& \leqq\left(\int_{T^{n}} \operatorname{Tr} S(x) \Psi(x) \Psi(x) S(x) d x\right)^{1 / 2}\left(\int_{T^{n}} \operatorname{Tr} \Phi(x) d x\right)^{1 / 2} \\
& =\left\langle S^{2}, \Phi\right\rangle^{1 / 2}\left(\int_{T^{n}} \operatorname{Tr} \Phi(x) d x\right)^{1 / 2} .
\end{aligned}
$$

It follows from (2.19) that

$$
\frac{d}{d t}\langle S, \Phi\rangle+\frac{\langle S, \Phi\rangle^{2}}{\int_{T^{n}} \operatorname{Tr} \Phi(x) d x} \leqq 0 .
$$

We assumed in (d) that $\left\langle S_{0}, \Phi\right\rangle\langle 0$ and thus we infer that $\langle S(t, \cdot), \Phi\rangle$ must become $-\infty$ for $t$ not larger than $T_{\infty}=\frac{\int_{T^{n}} \operatorname{Tr} \Phi(x) d x}{\left|\left\langle S_{0}, \Phi\right\rangle\right|}$.

We are going to show now how one can construct $\Phi$ satisfying properties (a)-(d). Let us take a neighborhood $V$ of $x_{0}$ such that for $y \in V, \Gamma_{i}(y) \cap \operatorname{supp} J_{0}=\emptyset$. Since $U_{0 i i}(x)=S_{0 i i}(x)$, assumption (ii) implies

$$
\int_{\Gamma_{i}\left(x_{0}\right)} S_{0 i i}\left(x_{01}, \ldots, x, \ldots, x_{0 n}\right) d x
$$


is negative. We may assume that

$$
\int_{\Gamma_{i}(y)} S_{0 i i}<0 \text { for all } y \in V .
$$

Let $\varphi(x)=\psi(x)^{2}$ with $\psi(x)$ a smooth function defined in $T^{n}$, independent of the $i^{\text {th }}$ variable (that is, constant on circles $\Gamma_{i}(z)$ for any $z \in T^{n}$ ) with support in $K=\bigcup_{y \in V} \Gamma_{i}(y)$, and identically 1 on a set $K_{1}=\bigcup_{y \in V_{1}} \Gamma_{i}(y)$ for some $x_{0} \in V_{1} \subset \subset V$. We define $\Phi(x)$ to be the $n \times n$ matrix having all entries equal to zero with the exception of the entry $\Phi(x)_{i i}$ set to be equal to $\varphi(x)$. Clearly properties (a), (b), and (d) are satisfied from construction. Condition (c) is satisfied for a matrix $\Phi$ if its columns are divergence free. In the constructed matrix the only nonzero column is the $i^{\text {th }}$ and $\partial_{i} \varphi(x)=0$. This completes the proof of Theorem 2.1.

\section{Solutions with Spherical Symmetry}

In [4] Foias showed that Eq. (2.10) in the whole space $\mathbb{R}^{n}$ admits solutions of the form $U(t, x)=\beta(t,|x|)(I-n \pi(x))$, where $I$ is the identity matrix and $\pi(x)$ is the projector on the direction $x$,

$$
\pi(x)=\left(\frac{x_{i} x_{j}}{|x|^{2}}\right) \quad i, j=1, \ldots, n .
$$

Moreover he obtained a simple equation for the scalar quantity $\beta$ which blows up. We shall generalize slightly this result, allowing antisymmetric parts in $U(t, x)$. The main reason behind our desire to have nontrivial antisymmetric parts in $U(t, x)$ is that they correspond to the vorticity in the case of genuine Euler equations.

Let $A$ be a rotation, $A \in O(n)$. We denote for a scalar function in $\mathbb{R}^{n}, f$, by $f_{A}$ the composed

$$
f_{A}(x)=f(A x)
$$

For a matrix valued function $M$ we denote by $M_{A}$ the matrix with entries $\left(M_{A}\right)_{i j}$ $=\left(M_{i j}\right)_{A}$. We define the operations $T_{A}$ and $\widetilde{T}_{A}$ on $n \times n$ matrix valued function as

$$
\begin{gathered}
T_{A} M=A^{-1} M_{A} A, \\
\widetilde{T}_{A} M=(\operatorname{det} A) A^{-1} M_{A} A .
\end{gathered}
$$

Finally, for a matrix valued function $U$ we define $L_{A}(U)$ by

$$
L_{A}(U)=T_{A}(S)+\widetilde{T}_{A}(J), \text { where } U=S+J,
$$

$S=\frac{1}{2}\left(U+U^{*}\right), J=\frac{1}{2}\left(U-U^{*}\right)$. Let us denote by $N(U)$ the operator giving the distorted Euler equation in $\mathbb{R}^{n}$ :

$$
N(U)=\partial_{t} U+U^{2}+R\left(\operatorname{Tr} U^{2}\right) .
$$

Proposition 3.1. For any $A \in O(n), N$ is covariant with respect to $L_{A}$ :

$$
L_{A}(N(U))=N\left(L_{A} U\right) .
$$


Corollary 3.2. If the initial data $U_{0}$ is invariant with respect to A, i.e. if $L_{A}\left(U_{0}\right)=U_{0}$, then the solution $U(t, x)$ is invariant with respect to $A$,

$$
L_{A}(U(t, \cdot))=U(t, \cdot) .
$$

Proof of Proposition 3.1. Let us take the symmetric and antisymmetric parts of $N(U)$,

$$
\begin{gathered}
\frac{N(U)+N(U)^{*}}{2}=\partial_{t} S+S^{2}+J^{2}+R\left(\operatorname{Tr} U^{2}\right), \\
\frac{N(U)-N(U)^{*}}{2}=\partial_{t} J+S J+J S .
\end{gathered}
$$

Applying $\tilde{T}_{A}$ to (3.10) we obtain

$$
\widetilde{T}_{A}\left(\frac{N(U)-N(U)^{*}}{2}\right)=\partial_{t} \widetilde{T}_{A} J+\left(T_{A} S\right)\left(\widetilde{T}_{A} J\right)+\left(\widetilde{T}_{A} J\right)\left(T_{A} S\right)=\frac{N\left(L_{A} U\right)-\left(N\left(L_{A} U\right)\right)^{*}}{2} .
$$

In order to check the $T_{A}$ covariance of the symmetric part of $N(U)$ we make use of the well-known covariance with respect to rotations of the Riesz transforms ([6])

for any scalar function $f$.

$$
T_{A}(R f)=R\left(f_{A}\right)
$$

We check now that $\operatorname{Tr}\left(L_{A} U\right)^{2}=\left(\operatorname{Tr} U^{2}\right)_{A}$. Indeed

$$
\begin{aligned}
\operatorname{Tr}\left(L_{A} U\right)^{2} & =\operatorname{Tr}\left(\left(T_{A} S+\widetilde{T}_{A} J\right)^{2}\right)=\operatorname{Tr}\left(\left(T_{A} S\right)^{2}+\left(\widetilde{T}_{A} J\right)^{2}\right)=\operatorname{Tr}\left(T_{A}\left(S^{2}\right)+T_{A}\left(J^{2}\right)\right) \\
& =\operatorname{Tr} A^{-1}\left(S^{2}+J^{2}\right)_{A} A=\operatorname{Tr}\left(S^{2}+J^{2}\right)_{A}=\left(\operatorname{Tr} U^{2}\right)_{A} .
\end{aligned}
$$

Applying $T_{A}$ to (3.9) we obtain

$$
\begin{aligned}
T_{A}\left(\frac{N(U)+N(U)^{*}}{2}\right) & =\partial_{t}\left(T_{A} S\right)+\left(T_{A} S\right)^{2}+\left(\widetilde{T}_{A} J\right)^{2}+R\left(\operatorname{Tr}\left(L_{A} U\right)^{2}\right) \\
& =\frac{N\left(L_{A} U\right)+\left(N\left(L_{A} U\right)\right)^{*}}{2} .
\end{aligned}
$$

This proves the proposition. Corollary (3.2) follows from uniqueness of solutions of $N(U)=0$.

Let us restrict our attention for a moment to the case $n=3$. Any antisymmetric matrix $J$ defines uniquely a vector $\omega \in \mathbb{R}^{3}$ such that $J v=\omega \times v$ for any $v \in \mathbb{R}^{3}$. Here $\omega \times v$ is the vector $\left(\omega_{2} v_{3}-\omega_{3} v_{2}, \omega_{3} v_{1}-\omega_{1} v_{3}, \omega_{1} v_{2}-\omega_{2} v_{1}\right)^{t}$ and clearly $\omega$ is determined by $\omega_{1}=J_{32}, \omega_{2}=J_{13}, \omega_{3}=J_{21}$. The matrix $J^{2}$ can be computed in terms of the vector $\omega$ :

where $\pi_{\omega}=\frac{\left(\omega_{i} \omega_{j}\right)}{|\omega|^{2}}$.

We note here that if $J(x)$ is the antisymmetric part of the Jacobian of a function $u(x)$, i.e. $J(x)=\frac{1}{2}\left(\partial_{j} u_{i}-\partial_{i} u_{j}\right), i, j=1,2,3$, then $\omega(x)=\frac{1}{2}(\nabla \times u)(x)$. If $J(t, x)$ satisfies the antisymmetric part of (2.10), i.e. if

$$
\partial_{t} J+S J+J S=0
$$


then forming the quantities $\omega(t, x)$ corresponding to $J(t, x)$, we obtain from (3.13) the equation

$$
\partial_{t} \omega=S \omega
$$

This is the analogue of the vorticity equation in the case of Euler equations. Summarizing, Eq. (2.10) is equivalent in the three-dimensional case to

$$
\partial_{t} S+S^{2}+J^{2}+R\left(\operatorname{Tr} S^{2}+\operatorname{Tr} J^{2}\right)=0
$$

coupled with (3.14), where $J^{2}$ is given by (3.12). We can consider the system (3.14), (3.15) with $J^{2}$ defined by (3.12) in any number of dimensions: $S$ will be a $n \times n$ symmetric matrix and $\omega$ an $n$ vector.

Proposition 3.2. Assume that the initial data for the system

$$
\begin{gathered}
\partial_{t} \omega=S \omega, \\
\partial_{t} S+S^{2}+J^{2}+R\left(\operatorname{Tr}\left(S^{2}+J^{2}\right)\right)=0,
\end{gathered}
$$

where $J^{2}=-|\omega|^{2}\left(I-\pi_{\omega}\right), \pi_{\omega}=\left(\frac{\omega_{i} \omega_{j}}{|\omega|^{2}}\right), i, j=1, \ldots, n$ are of the form

$$
\begin{gathered}
\omega_{0}(x)=\gamma_{0}(|x|) \cdot \frac{x}{|x|}, \quad x \in \mathbb{R}^{n}, \\
S_{0}(x)=\beta_{0}(|x|)(I-n \pi(x)), \pi(x) \text { given in }(3.1) .
\end{gathered}
$$

Then for as long as the solution $S(t, x), \omega(t, x)$ stays smooth, it has the form

$$
\begin{gathered}
\omega(t, x)=\gamma(t,|x|) \frac{x}{|x|}, \\
S(t, x)=\beta(t,|x|)(I-n \pi(x)),
\end{gathered}
$$

where $\gamma, \beta$ are two scalar functions satisfying

$$
\begin{gathered}
\partial_{t} \beta+\beta^{2}-\gamma^{2}-\frac{n-1}{r^{n}} \int_{0}^{r} s^{n-1}\left(n \beta^{2}-\gamma^{2}\right) d s=0, \\
\partial_{t} \gamma+(n-1) \gamma \beta=0, \\
\gamma(0, r)=\gamma_{0}(r), \quad \beta(0, r)=\beta_{0}(r), \\
\gamma(t, 0)=\gamma(t, \infty)=0, \quad \beta(t, 0)=\beta(t, \infty)=0 .
\end{gathered}
$$

Remark 1 . The equation obtained by Foias is the particular case $\gamma(t, r) \equiv 0$ arising from $\gamma_{0} \equiv 0$.

Remark 2. In $n=3$ initial data of the form (3.18), (3.19) are those which satisfy $L_{A} U_{0}=U_{0}$, for all $A \in O(3), \operatorname{Tr} U_{0}=0$.

We start by computing $R f$ for a radial function.

Lemma 3.3. Let $f=f(r)$ be a smooth function defined for $r \geqq 0$ decaying sufficiently at infinity ( for instance $f(|x|) \in L^{1} \cap L^{2}$ in $\mathbb{R}^{n}$ ). Then

$$
R f=-[g I+k \pi],
$$


where $g$ and $k$ are radial functions defined by

$$
\begin{gathered}
g(r)=\frac{1}{r^{n}} \int_{0}^{r} s^{n-1} f(s) d s, \\
k(r)=f(r)-\frac{n}{r^{n}} \int_{0}^{r} s^{n-1} f(s) d s .
\end{gathered}
$$

Proof. Let us use the notation $f^{\prime}=\frac{d f}{d r}, r=|x|$. Then

$$
\partial_{i j}^{2} f(r)=\frac{f^{\prime}(r)}{r} \delta_{i j}+\left(\left(\frac{f^{\prime}(r)}{r}\right)^{\prime} r\right) \pi(x) \text {. }
$$

On the other hand

$$
\Delta\left(g(r) \delta_{i j}+h(r) x_{i} x_{j}\right)=(\Delta g+2 h) \delta_{i j}+\left(\Delta h+\frac{4 h^{\prime}}{r}\right) x_{i} x_{j} .
$$

Thus $\partial_{i j}^{2} f(r)=\Delta\left(g(r) \delta_{i j}+h(r) x_{i} x_{j}\right)$ if the system

$$
\begin{gathered}
\Delta g+2 h=\frac{f^{\prime}(r)}{r}, \\
r\left(\Delta h+\frac{4 h^{\prime}}{r}\right)=\left(\frac{f^{\prime}(r)}{r}\right)^{\prime} \text { is solved. }
\end{gathered}
$$

Now (3.30) follows from (3.29) if

$$
(\Delta g)^{\prime}+2 h^{\prime}=r\left(\Delta h+\frac{4 h^{\prime}}{r}\right), \quad \text { i.e. if }(\Delta g)^{\prime}=\left(r h^{\prime}\right)^{\prime}+n h^{\prime} .
$$

This follows if $\Delta g=(r h)^{\prime}+\frac{n-1}{r}(r h)$. So (3.30) is a consequence of (3.29) if $g^{\prime}=r h$. With this choice for $g$ we solve (3.29):

$$
(r h)^{\prime}+\frac{n-1}{r} r h+2 h=\frac{f^{\prime}}{r} .
$$

This gives $\left(r^{n} k\right)^{\prime}=r^{n} f^{\prime}$ for $k=r^{2} h$. We obtain the formula (3.28) for $k$ :

$$
k(r)=f(r)-\frac{n}{r^{n}} \int_{0}^{r} s^{n-1} f(s) d s .
$$

Then $g^{\prime}=\frac{1}{r} k$. In order to check (3.27), let us note that

$$
(n g+k-f)^{\prime}=\frac{n}{r} k+k^{\prime}-f^{\prime}=0 .
$$

Thus, since all these functions vanish at infinity we obtain

$$
f=n g+k \text {. }
$$


Therefore (3.31) and (3.32) imply (3.27). We note that (3.32) follows also from the familiar $R_{i} R_{i} f=-f$ (see [6]) by taking the trace in (3.26).

Proof of Proposition 3.2. We shall use the ansatz $\omega(t, x)=\gamma(t,|x|) \frac{x}{|x|}, S(t, x)$ $=\beta(t,|x|)(I-n \pi(x))$ and check that Eqs. (3.16), (3.17) give consistent equations for $\beta, \gamma$. Equation (3.16) becomes $\partial_{t} \gamma=(1-n) \beta \gamma$, i.e. (3.23). Now $S^{2}=\beta^{2}(I-n \pi)^{2}$ $=\beta^{2}\left(I+\left(n^{2}-2 n\right) \pi\right)$ because $\pi^{2}=\pi$. Also $J^{2}=-\gamma^{2}(I-\pi)$. Indeed $\pi_{\omega}=\pi(x)$ because $x$ and $\omega$ define the same direction. In order to proceed we put $f(r)$ $=\operatorname{Tr}\left(S^{2}+J^{2}\right)$, and compute

$$
\operatorname{Tr}\left(S^{2}+J^{2}\right)=f(r)=(n-1)\left[n \beta^{2}-\gamma^{2}\right] .
$$

According to Lemma 3.3 it follows that

$$
R\left(\operatorname{Tr}\left(S^{2}+J^{2}\right)\right)=-(g I+k \pi)
$$

with $g, k$ defined by (3.27), (3.28) and $f$ by (3.33). At this point Eq. (3.17) has the form

$$
\left(\partial_{t} \beta\right)(I-n \pi)+\left(\beta^{2}-\gamma^{2}-g\right) I+\left(\left(n^{2}-2 n\right) \beta^{2}+\gamma^{2}-k\right) \pi=0 .
$$

The only way in which (3.35) can possibly give a consistent equation for $\beta$ is if it factors out $(I-n \pi)$, that is if

$$
\left(n^{2}-2 n\right) \beta^{2}+\gamma^{2}-k=-n\left(\beta^{2}-\gamma^{2}-g\right) .
$$

But (3.36) is equivalent to

$$
(n-1)\left(n \beta^{2}-\gamma^{2}\right)=n g+k,
$$

which in view of (3.33) is nothing but (3.32) in disguise. Therefore Eq. (3.35) becomes

$$
\left(\partial_{t} \beta+\beta^{2}-\gamma^{2}-g\right)(I-n \pi)=0,
$$

which is satisfied if $\beta$ solves (3.22) because (3.27) and (3.33) imply

$$
g(r)=\frac{n-1}{r^{n}} \int_{0}^{r} s^{n-1}\left(n \beta^{2}-\gamma^{2}\right) d s
$$

We present now the blow-up argument.

Theorem 3.4. Let us assume that beside the conditions (3.18) and (3.19) of Proposition 3.2 being fulfilled, the initial data for the system (3.16), (3.17) satisfy also

$$
\begin{gathered}
\gamma_{0}(r)=0 \quad \text { for } \quad 0 \leqq r \leqq R_{1} \text { for some } R_{1}>0, \\
\beta_{0}(r)=0 \text { for } 0 \leqq r \leqq R \text { for some } 0 \leqq R<R_{1}, \\
\int_{R}^{\alpha R} \beta_{0}(r) r^{n-1} d r<0 \text { for some } 1<\alpha,(\alpha \text { near } 1) .
\end{gathered}
$$

Then the solution to the distorted Euler equations (3.16), (3.17) having

$$
\omega_{0}(x)=\gamma_{0}(|x|) \frac{x}{|x|}, \quad S_{0}(x)=\beta_{0}(|x|)(I-n \pi(x))
$$


for initial data breakdown in finite time. More precisely $S$ breaks down near $|x|=R$. Proof. As proven in Proposition (3.2) the solutions are $\omega(t, x)=\gamma(t,|x|) \frac{x}{|x|}, S(t, x)$ $=\beta(t,|x|)(I-n \pi)$ with $\gamma, \omega$ satisfying (3.22)-(3.25). Since (3.23) can be integrated,

$$
\gamma(t, r)=\gamma_{0}(r) \exp \int_{0}^{t}(1-n) \beta(s, r) d s
$$

it follows from (3.38) that

$$
\gamma(t, r)=0 \text { for } 0 \leqq r \leqq R_{1} .
$$

Therefore, for $0 \leqq r \leqq R_{1}$, Eq. (3.22) becomes

$$
\partial_{t} \beta+\beta^{2}-\frac{n-1}{r^{n}} \int_{0}^{r} s^{n-1} n \beta^{2}(s) d s=0 .
$$

Now we claim that from (3.43) it follows that property (3.39) is preserved by $\beta(t, r)$, $t \geqq 0$, as long as both $\beta$ and $\gamma$ are smooth. Indeed multiplying (3.43) by $\beta(t, r) r^{n-1}$, and integrating between 0 and $R$ one obtains

$$
\frac{1}{2} \frac{d}{d t} \int_{0}^{R} \beta^{2}(t, r) r^{n-1} d r+\int_{0}^{R} \beta^{3}(t, r) r^{n-1} d r=(n-1) n \int_{0}^{R} \beta^{2}(t, \varrho) \varrho^{n-1} \int_{\varrho}^{R} \frac{\beta(t, r)}{r} d r
$$

or

$$
\frac{1}{2} \frac{d}{d t} \int_{0}^{R} \beta^{2}(t, r) r^{n-1} d r=\int_{0}^{R} \beta^{2}(t, r) r^{n-1}\left[n(n-1) \int_{r}^{R} \frac{\beta(t, \varrho)}{\varrho} d \varrho-\beta(t, r)\right] d r .
$$

Thus

$$
\frac{d}{d t} \int_{0}^{R} \beta^{2}(t, r) r^{n-1} d r \leqq 2 \operatorname{Max}_{\varrho \in[0, R]}\left(n(n-1) \int_{\varrho}^{R} \frac{\beta(t, s)}{s} d s-\beta(t, \varrho)\right) \int_{0}^{R} \beta^{2}(t, r) r^{n-1} d r,
$$

and therefore, as long as $\beta$ is smooth,

$$
\int_{0}^{R} \beta^{2}(t, r) r^{n-1} d r \leqq \int_{0}^{R} \beta_{0}^{2}(r) r^{n-1} d r \exp 2 \int_{0}^{t} \operatorname{Max}_{\varrho \in[0, R]}\left(n(n-1) \int_{\varrho}^{R} \frac{\beta(\tau, s)}{s} d s-\beta(\tau, \varrho)\right) d \tau,
$$

and by (3.39) it follows

$$
\int_{0}^{R} \beta^{2}(t, r) r^{n-1} d r=0, \quad t \geqq 0 .
$$

Let us take now $\alpha>1$ such that (3.40) is valid and $\alpha$ small enough such that $\alpha R<R_{1}, n(n-1) \log \alpha<1$. Integrating in (3.43) between $R$ and $\alpha R$ we obtain

$$
\begin{aligned}
& \frac{d}{d t} \int_{R}^{\alpha R} \beta(t, r) r^{n-1} d r+(1-n(n-1) \log \alpha) \int_{R}^{\alpha R} \beta^{2}(t, s) s^{n-1} d s \\
& \quad \leqq n(n-1) \log \alpha \int_{0}^{R} \beta^{2}(t, s) s^{n-1} d s=0 .
\end{aligned}
$$

Now

$$
\int_{R}^{\alpha R} \beta^{2}(t, s) s^{n-1} d s \geqq \frac{n}{R^{n}} \frac{1}{\alpha^{n}-1}\left(\int_{R}^{\alpha R} \beta(t, s) s^{n-1} d s\right)^{2} .
$$


It follows that

$$
\frac{d}{d t} \int_{R}^{\alpha R} \beta(t, r) r^{n-1} d r+\frac{n(1-n(n-1) \log \alpha)}{R^{n}\left(\alpha^{n}-1\right)}\left(\int_{R}^{\alpha R} \beta(t, s) s^{n-1} d s\right)^{2} \leqq 0,
$$

and since

$$
\int_{R}^{\alpha R} \beta_{0}(r) r^{n-1} d r<0
$$

we conclude that $\int_{R}^{\alpha R} \beta(t, r) r^{n-1} d r$ becomes $-\infty$ for $t$ not larger than

$$
T_{\infty}=\frac{R^{n}\left(\alpha^{n}-1\right)}{n(1-n(n-1) \log \alpha)} \cdot \frac{1}{\left|\int_{R}^{\alpha R} \beta_{0}(r) r^{n-1} d r\right|} .
$$

One can easily obtain a blow-up argument for Eqs. (3.22), (3.23) at the origin if one drops the requirement that $\beta_{0}(0)=0$. However, this would lead to functions $S(t, x)$ which are not defined at $x=0$.

Acknowledgements. It is a pleasure to thank A. Majda for suggesting the result of Sect. 1 and for stimulating discussions. I had many interesting conversations with C. Foias and S. Klainerman. It is an equal pleasure to thank them both.

\section{References}

1. Beale, J.T., Kato, T., Majda, A.: Remarks on the breakdown of smooth solutions for the $3-D$ Euler equations. Commun. Math. Phys. 94, 61-66 (1984)

2. Constantin, P.: Blow-up for a non-local evolution equation. M.S.R.I. 038-84-6 Berkeley, California, July 1984

3. Constantin, P., Lax, P.D., Majda, A.: A simple one dimensional model for the three dimensional vorticity equation (submitted to Commun. Pure Appl. Math.)

4. Foias, C.: Unpublished

5. Kato, T.: Nonstationary flows of viscous and ideal fluids in $\mathbb{R}^{3}$. J. Funct. Anal.9, 296-305 (1972)

6. Stein, E.M., Weiss, G.: Introduction to Fourier analysis on Euclidean spaces. Princeton, NJ: Princeton University Press 1971

7. Swann, H.: The convergence with vanishing viscosity of nonstationary Navier-Stokes flow to ideal flow in $\mathbb{R}^{3}$. Trans. Am. Math. Soc. 157, 373-397 (1971)

Communicated by A. Jaffe

Received November 12, 1985 